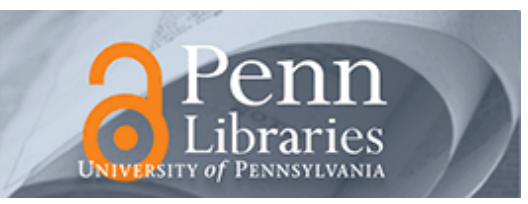

University of Pennsylvania

ScholarlyCommons

\title{
Serious discrepancies between nuclear magnetic resonance and neutron scattering measurements in the critical region of classic two-dimensional magnets and anomalous behaviors at low temperatures
}

Nicholas Bykovetz

Temple University

J. Klein

University of Pennsylvania

C. L. Lin

Temple University

Follow this and additional works at: https://repository.upenn.edu/physics_papers

Part of the Physics Commons

\section{Recommended Citation}

Bykovetz, N., Klein, J., \& Lin, C. L. (2011). Serious discrepancies between nuclear magnetic resonance and neutron scattering measurements in the critical region of classic two-dimensional magnets and anomalous behaviors at low temperatures. Retrieved from https://repository.upenn.edu/physics_papers/ 136

Suggested Citation:

Bykovetz, N., Klein, J. and Lin, C.L. (2007). Serious discrepancies between nuclear magnetic resonance and neutron scattering measurements in the critical region of classic two-dimensional magnets and anomalous behaviors at low temperatures. Journal of Applied Physics. 109, 07 E119.

(C) 2007 American Institute of Physics. This article may be downloaded for personal use only. Any other use requires prior permission of the author and the American Institute of Physics. The following article appeared in Journal of Applied Physics and may be found at http://dx.doi.org/10.1063/1.3549596.

This paper is posted at ScholarlyCommons. https://repository.upenn.edu/physics_papers/136

For more information, please contact repository@pobox.upenn.edu. 


\title{
Serious discrepancies between nuclear magnetic resonance and neutron scattering measurements in the critical region of classic two-dimensional magnets and anomalous behaviors at low temperatures
}

\author{
Abstract \\ A characterization of 2D critical behavior in the classic $2 \mathrm{D}$ antiferromagnets $\mathrm{K}_{2} \mathrm{NiF}_{4}, \mathrm{Rb}_{2} \mathrm{MnF}_{4}$, and \\ $\mathrm{K}_{2} \mathrm{MnF}_{4}$ made originally by Birgeneau et al. [Phys. Rev. B 1, 2211 (1970)], using neutron scattering (NS), is \\ used to review NMR data in these compounds and in some classic 2D ferromagnets. On analyzing the \\ NMR data, very serious discrepancies were found between the NMR and NS results for the critical \\ exponent $B$, with the values of the NMR ßs being some $50 \%$ higher. Some novel results were also found, \\ including $2 D$ cases where $B=0.33$ (but characterized by a non-3D parameter, $D \sim 1$ ). The discrepancies \\ surely need to be resolved, and if the present analysis of the NMR data is further validated, these results \\ could present a clear and nontrivial hurdle for current critical-region theories. Additionally, from the NMR \\ data it was found that the low-temperature behaviors of these classical 2D systems are at odds with the \\ predictions of conventional spin-wave theory analyses.

\section{Disciplines} \\ Physical Sciences and Mathematics | Physics

\section{Comments} \\ Suggested Citation: \\ Bykovetz, N., Klein, J. and Lin, C.L. (2007). Serious discrepancies between nuclear magnetic resonance \\ and neutron scattering measurements in the critical region of classic two-dimensional magnets and \\ anomalous behaviors at low temperatures. Journal of Applied Physics. 109, $07 \mathrm{E} 119$. \\ (C) 2007 American Institute of Physics. This article may be downloaded for personal use only. Any other \\ use requires prior permission of the author and the American Institute of Physics. The following article \\ appeared in Journal of Applied Physics and may be found at http://dx.doi.org/10.1063/1.3549596.
}




\title{
Serious discrepancies between nuclear magnetic resonance and neutron scattering measurements in the critical region of classic two-dimensional magnets and anomalous behaviors at low temperatures
}

\author{
N. Bykovetz, ${ }^{1}$ J. Klein, ${ }^{2}$ and C. L. Lin ${ }^{1, a)}$ \\ ${ }^{1}$ Department of Physics, Temple University, Philadelphia, Pennsylvania 19122, USA \\ ${ }^{2}$ Department of Physics and Astronomy, University of Pennsylvania, Philadelphia, Pennsylvania 19104-6396, \\ USA
}

(Presented 16 November 2010; received 1 October 2010; accepted 14 November 2010; published online 25 March 2011)

\begin{abstract}
A characterization of $2 \mathrm{D}$ critical behavior in the classic $2 \mathrm{D}$ antiferromagnets $\mathrm{K}_{2} \mathrm{NiF}_{4}, \mathrm{Rb}_{2} \mathrm{MnF}_{4}$, and $\mathrm{K}_{2} \mathrm{MnF}_{4}$ made originally by Birgeneau et al. [Phys. Rev. B 1, 2211 (1970)], using neutron scattering (NS), is used to review NMR data in these compounds and in some classic 2D ferromagnets. On analyzing the NMR data, very serious discrepancies were found between the NMR and NS results for the critical exponent $\beta$, with the values of the NMR $\beta$ s being some $50 \%$ higher. Some novel results were also found, including $2 \mathrm{D}$ cases where $\beta=0.33$ (but characterized by a non-3D parameter, $D \sim 1$ ). The discrepancies surely need to be resolved, and if the present analysis of the NMR data is further validated, these results could present a clear and nontrivial hurdle for current critical-region theories. Additionally, from the NMR data it was found that the low-temperature behaviors of these classical 2D systems are at odds with the predictions of conventional spin-wave theory analyses. (c) 2011 American Institute of Physics. [doi:10.1063/1.3549596]
\end{abstract}

\section{INTRODUCTION}

Neutron (as well as other studies) of the classic quadratic $2 \mathrm{D}$ antiferromagnets $\mathrm{K}_{2} \mathrm{NiF}_{4}, \mathrm{Rb}_{2} \mathrm{MnF}_{4}$, and $\mathrm{K}_{2} \mathrm{MnF}_{4}$ were instrumental in providing support for the current theory of critical phenomena. In particular, the neutron scattering (NS) measurements of Birgeneau et al. ${ }^{1}$ determined that the values of the critical exponent $\beta$ in these systems were very close to the exact 2D Ising model value of $1 / 8$. In connection with unrelated issues, we were recently led to reexamine the results of Ref. 1, as well as other determinations of $\beta$ in 2D magnetic systems, and found strong evidence that the neutron results may be seriously in error. Extending this analysis to $2 \mathrm{D}$ ferromagnets gave additional indications that our findings may actually pose substantial empirical hurdles for current critical theory.

Birgeneau et al. ${ }^{1}$ made the very interesting observation that the sublattice magnetization of $\mathrm{K}_{2} \mathrm{NiF}_{4}$ and $\mathrm{Rb}_{2} \mathrm{MnF}_{4}$ when fitted to the usual critical region power-law equation, $M / M_{0}=D\left(1-T / T_{c}\right)^{\beta}$, followed the magnetization curve all the way up to $\sim 90 \% M_{0}$ (unlike $3 \mathrm{D}$ counterparts), and even on the low-temperature side deviated only slightly from the measured magnetization curve. The critical region equation coincides with the magnetization curve exactly up to $\sim 0.90 M_{0}$, and then still hugs it closely up to $T=0$. This result is characterized by the value of $D$ being close to 1.0, instead of some $20 \%$ higher, as in the case of $3 \mathrm{D}$ magnets and the $2 \mathrm{D}$ Ising model. We will refer to these specifications of the $2 \mathrm{D}$ critical behavior as the Birgeneau et al. Critical Region Characterization (BCRC).

\footnotetext{
a) Author to whom correspondence should be addressed. Electronic mail: clin@temple.edu.
}

We have examined a number of systems in which the magnetization (or sublattice magnetization) of 2D systems was measured with fairly good precision and found that indeed the BCRC criterion appears to hold. Since the exquisitely beautiful and precise NMR data of de Wijn et al. ${ }^{2}$ on the classic 2D systems were available to us in numerical form, ${ }^{3}$ we examined them for consistency with the neutron data of Ref. 1 using the BCRC criterion as a guide, and were quite surprised to find unexpected serious discrepancies.

\section{RESULTS AND DISCUSSION}

Our analysis of the NMR data of $\mathrm{K}_{2} \mathrm{NiF}_{4}$ shows an excellent power-law behavior, but gave a $\beta=0.20$, instead of 0.14 (from NS). For both $\mathrm{K}_{2} \mathrm{MnF}_{4}$ and $\mathrm{Rb}_{2} \mathrm{MnF}_{4}$ the fitted $\beta=0.25$ (instead of 0.15 and 0.16 , respectively). Fits of the NMR data for the double-layered quasi-2D system $\mathrm{K}_{3} \mathrm{Mn}_{2} \mathrm{~F}_{7},{ }^{4}$ likewise showed an excellent power-law fit and also produced a $\beta=0.25, D=1.06$, in contrast to the NS $\beta=0.154$ and $D=0.83$. $^{5}$ In all of these compounds we found $D \sim 1.06$.

Figure 1 displays the NMR data for $\mathrm{K}_{2} \mathrm{NiF}_{4}$, showing a plot of Freq ${ }^{5}$ vs $T$. One can see how precise the NMR data are, in contrast to the much larger scatter of data points displayed in essentially all NS measurements of this kind. Since there is not the slightest inkling of deviation from a straight line in Fig. 1, as one approaches $T_{c}$, and the fitted $T_{c}$ of 99.5 $\mathrm{K}$, though a bit higher than the NS $T_{c}$, agrees quite well with the values reported from other techniques (100.5 K from NMR linewidths and 98.7 from specific heat), it is not likely that a different $\beta$ would be found in the last $\sim 8 \%$ of the pre$T_{c}$ region. But, if it were to be the case that the $\beta$ changed in $0.92<T / T_{c}<1.00$, then there is still a very significant 
discrepancy between the NS and NMR results for $T / T_{c}<0.92$, and so the measurements are still without doubt in disagreement. Since the fluorine resonance is exceptionally narrow, and the thermometric procedures described in Ref. 2 are superb, we believe it is the NS data that are giving erroneous results. While we will not try to pinpoint the actual problem in the NS measurements, it seems likely that extinction effects may be responsible.

The presentations of NS data do not normally indicate how the fitted parameters are obtained, but presumably it is directly from the equation $M / M_{0}=D\left(1-T / T_{c}\right)^{\beta}$. The graphical presentations of the data are normally shown on a $\log -\log$ plot. We prefer to restructure this equation in the form of a simple power-law, $y=A+B X^{C}$, both for obtaining the fitted parameters as well as for the graphic portrayal of the critical region. In this format, the critical equation takes the form $T=T_{c}+B M^{1 / \beta}$, with the fitted parameters being the exponent $1 / \beta, T_{c}$, and the slope $B$, from which $D$ can be extracted (cf., Fig. 1, where we have plotted $T$ on the horizontal axis for conformity with convention). The virtue of this form is that the exponent (here, $1 / \beta$ ) is determined entirely by the curvature of the data points (one obtains a straight line only for the correct exponent), so that graphs of the data using various exponents give a good feel for how much uncertainty (possible error) is truly consistent with the actual data, while analytical least-squares fitting can often give a misleading impression. We believe the latter may frequently be the case in NS analyses of the critical region. In particular, the portrayal of the data on a log-log plot can be misleading because the $x$-axis variable is intertwined with one of the fitting parameters $\left(T_{c}\right)$, so that when a crossover in $\beta$ is alleged to take place, the $\log -\log$ plots make it difficult to see whether an improper choice of $T_{c}$ is responsible, or an actual change in $\beta$.

Using the above-mentioned fitting procedures and graphical analysis and portrayals, we next examined the extensively studied classic $2 \mathrm{D}$ ferromagnets $\mathrm{K}_{2} \mathrm{CuF}_{4}$ and $\mathrm{CrCl}_{3}$. Only $\mathrm{K}_{2} \mathrm{CuF}_{4}$ was studied by NS as well as NMR, but here again the discrepancy between the NMR and NS results are significant. Our analysis indicates that both of these systems (as well as a number of other similar systems) show a BCRC $\beta=1 / 3$ in the range $0.9 M_{0}>M>0$. While values of $\beta$ near $1 / 3$ previously have been noted for $2 \mathrm{D}$ systems under some conditions, they were invariably discounted as an indi-

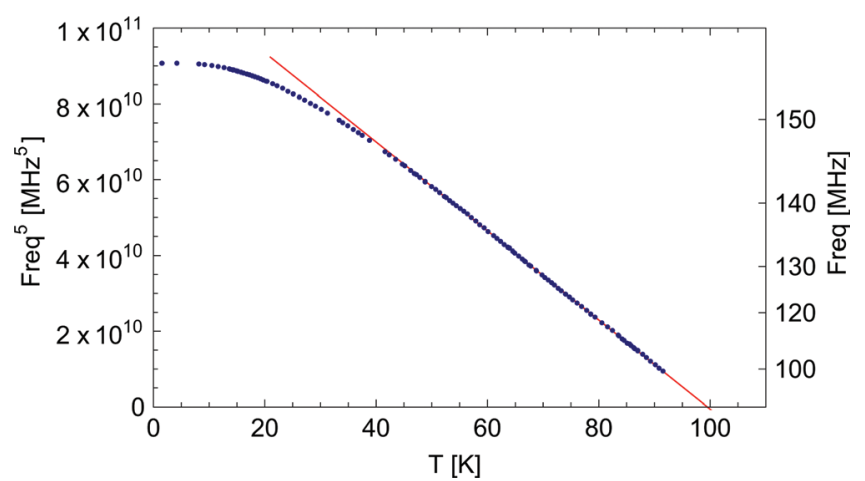

FIG. 1. (Color online) NMR data of de Wijn et al. (Ref. 2) for $\mathrm{K}_{2} \mathrm{NiF}_{4}$ plotted as Freq ${ }^{5}$ vs $T$ to display the BCRC critical region behavior. cation of a crossover to 3D critical behavior. We believe our analysis shows that the $\beta$ of $1 / 3$, accompanied by a $D$ close to 1 (as opposed to $\sim 1.20$ ), is a pure $2 \mathrm{D}$ characteristic of the critical behavior of certain $2 \mathrm{D}$ systems. It is viewed as a problem only insofar as being contrary to current criticalregion theory, which disallows a universal $2 \mathrm{D}$ exponent of $1 / 3$. While a recent review article by Taroni et al. ${ }^{6}$ does point out that nonuniversal exponents in the 2D $X Y$ model with fourfold crystal field can take on an unbounded spectrum of $\beta$ values, this model has no special preference for $\beta=1 / 3$.

In support of the above-presented view, we present the BCRC analysis of the $\mathrm{K}_{2} \mathrm{CuF}_{4} \mathrm{NMR}$ of Kubo et al. ${ }^{7}$ The data extend over $0.25<T / T_{c}<0.65$, and as can be seen from Fig. 2, fit to a $1 / \beta$ exponent of 3 very well (with a derived $D$ value of 1.06). While the $\mathrm{Cu}$ resonance data are not quite as precise as the data of Ref. 2, and a least-squares fit gives a $1 / \beta$ of $2.86 \pm 0.07$ (not exactly 3 ), the Kubo et al. data are clearly incompatible with the Hirakawa and Ikeda [(HI), Ref. 8] NS $\beta$ value of $0.22(1 / \beta=4.5)$, contrary to their explicit statements. HI inelastic NS measurements on $\mathrm{K}_{2} \mathrm{CuF}_{4}$ clearly show that this system displays $2 \mathrm{D}$ behavior at temperatures below, as well as above, $T_{c}$. HI claim their NS magnetization measurements cross over to $3 \mathrm{D}$ behavior with $\beta=0.33$ in the region $0.02>1-\left(T / T_{c}\right)>0.001$. Faraday rotation (FR) measurements ${ }^{9}$ show that $\beta=0.33$ holds over a much wider range than HI's, near $T_{c}$. Our conclusion from the NMR and the FR measurements, taken together, is that $\beta=0.33$ over the entire magnetization curve up to $0.9 M_{0}$. Thus, there appears to be no real evidence for a dimensionality crossover in $\mathrm{K}_{2} \mathrm{CuF}_{4}$, but as in the fluoride systems, there does appear to be a really serious discrepancy between the results of NS and other measurements.

$\mathrm{CrCl}_{3}$ is another classic 2D ferromagnet. No NS measurements were made, but the ${ }^{53} \mathrm{Cr}$ NMR data of Narath and Davis, ${ }^{10}$ like the NMR data of the fluoride compounds, are exceptionally precise, and performed with outstanding thermometry. In the low-temperature regime $\left(M_{0}>M>0.9 M_{0}\right)$, i.e., for $T<4 \mathrm{~K}, \mathrm{CrCl}_{3}$ exhibits a precise $T^{1.33}$ fall-off of the magnetization. For $\left(M<0.9 M_{0}\right)$, i.e., for $T>4 \mathrm{~K}$, the magnetization can be fitted to the exact same $T^{3 / 2}$ fall-off as reported by Kubo et al., ${ }^{7}$ for $\mathrm{K}_{2} \mathrm{CuF}_{4}$, in what we will call the "intermediate region" of the magnetization curve. As in the case of $\mathrm{K}_{2} \mathrm{CuF}_{4}$, we find that this translates into a BCRC critical-region fit of $\beta=1 / 3$, with a $D=1.06$.

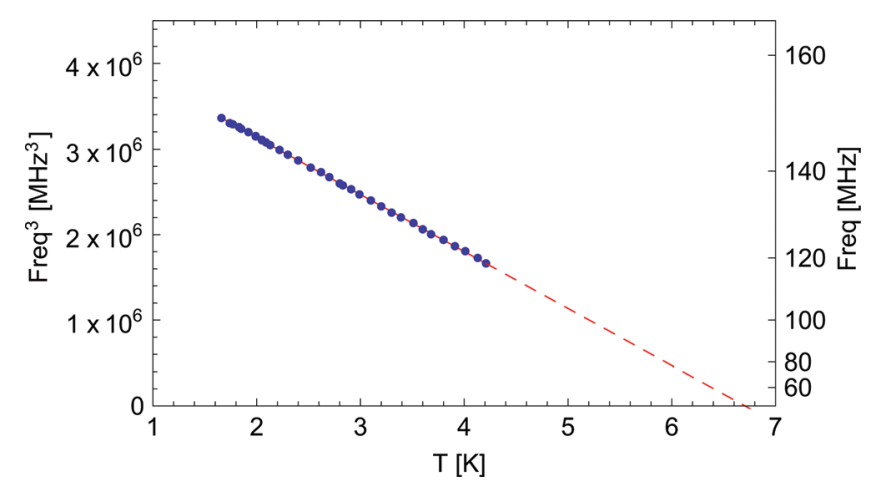

FIG. 2. (Color online) NMR data of Kubo et al. (Ref. 7) for $\mathrm{K}_{2} \mathrm{CuF}_{4}$ plotted as Freq $^{3}$ vs $T$ to display the BCRC critical region behavior. 
TABLE I. 2D critical region parameters from NMR.

\begin{tabular}{lccr}
\hline \hline Magnet & $\beta$ & $D$ & $T_{c}(\mathrm{~K})$ \\
\hline $\mathrm{K}_{2} \mathrm{NiF}_{4}$ & 0.20 & 1.05 & 99.5 \\
$\mathrm{~K}_{2} \mathrm{MnF}_{4}$ & 0.25 & 1.06 & 43.7 \\
$\mathrm{Rb}_{2} \mathrm{MnF}_{4}$ & 0.25 & 1.06 & 39.4 \\
$\mathrm{~K}_{3} \mathrm{Mn}_{2} \mathrm{~F}_{7}$ & 0.25 & 1.06 & 57.4 \\
$\mathrm{~K}_{2} \mathrm{CuF}_{4}$ & 0.33 & 1.06 & 6.7 \\
$\mathrm{CrCl}_{3}$ & 0.33 & 1.06 & 15.1 \\
\hline \hline
\end{tabular}

Similar results appear to hold in the 2D systems $\left(\mathrm{C}_{n} \mathrm{H}_{2 n+1} \mathrm{NH}_{3}\right)_{2} \mathrm{CuCl}_{4}$, which show an intermediate region magnetization fall-off proportional to $T^{3 / 2}$, as reported by Kubo et al. ${ }^{11}$ Although NMR data in numerical form were unavailable for these compounds, we have no doubt that the BCRC fits will also produce a $\beta=1 / 3$, since both $\mathrm{K}_{2} \mathrm{CuF}_{4}$ and $\mathrm{CrCl}_{3}$ behave in an identical manner. De Jongh et al. ${ }^{12}$ made an indirect measurement of the entire magnetization curve of $\left(\mathrm{C}_{n} \mathrm{H}_{2 n+1} \mathrm{NH}_{3}\right)_{2} \mathrm{CuCl}_{4}$ and found to their stated surprise that an excellent fit was obtained to the critical equation across the entire measured region, giving $\beta=0.33 \pm 0.02$, and $D=1.08 \pm 0.08$.

Summing up, 2D planar (spin-aligned-in-plane) ferromagnets all appear to show an exponent $\beta \sim 0.33$, but with a $D$ near 1.0 . We note that $\mathrm{CrCl}_{3}$, unlike the other $2 \mathrm{D}$ ferromagnetic systems, cannot be considered to be an $X Y$ system. Thus, the $2 \mathrm{D}$ ferromagnetic $\beta=1 / 3$ with $D \sim 1$ feature cannot be a characteristic of alleged $X Y$ systems alone, but must be more universal.

The various results for the BCRC critical behaviors of the classic 2D systems are summarized in Table I.

We tentatively note that the BCRC results obtained for the classical 2D magnets suggest that the only correct values of $1 / \beta$ may be the integers, specifically, 3 , 4 , and 5 (although we cannot exclude 6). If so, these integers could stem from different classes of partition functions, and not from general principles of universality, scaling, relevant parameters, etc., as in current critical theory. An example of what we mean is given in the simple imaginary-constraint spin system worked out by Ben-Abraham and Nudelman. ${ }^{13}$ It should also be remembered that the $1 / \beta 2 \mathrm{D}$ Ising model exponent of 8 was obtained directly from the partition function, and not from Renormalization Group theory.

The low-temperature behaviors of the above-mentioned systems are also of great interest, because they are at odds with conventional spin-wave theory (CSWT). Both the layered fluorine compounds, as well $\mathrm{CrCl}_{3}$, display simple power-law behaviors at low temperatures (i.e., in the region $\left.M>0.90 M_{0}\right)$, in the form $\mathrm{M} / \mathrm{M}_{0}=\mathrm{A}+\mathrm{BT}^{\mathrm{c}}$, not consonant with CSWT. In $\mathrm{CrCl}_{3}$, we find the exponent to be $4 / 3$, whereas all of the four fluoride compounds exhibit a $\mathrm{c}=2$. In Fig. 3 we show the $\mathrm{T}^{2}$ behavior of $\mathrm{K}_{2} \mathrm{NiF}_{4}$ for $\mathrm{T} / \mathrm{T}_{\mathrm{c}}<$

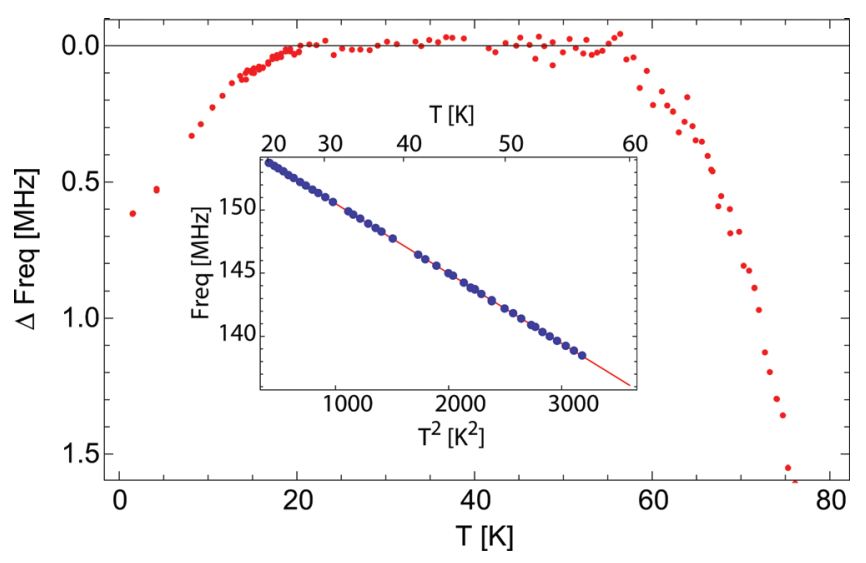

FIG. 3. (Color online) Low-temperature $T^{2}$ behavior of $\mathrm{K}_{2} \mathrm{NiF}_{4}$ for $20 \mathrm{~K}<T<56 \mathrm{~K}$. Deviations show the excellent fit to $T^{2}$, and deviations below $\sim 0.7 T_{\text {gap }}$, and at the onset of the critical region, $\sim 0.5 T_{c}$.

$\sim 0.5 \mathrm{~T}_{\mathrm{c}}$. The insert shows the spectacular fit to a straight line.

These simple low-temperature behaviors of the magnetization are clearly at odds with the CSWT analyses. ${ }^{2,4,10}$ Bykovetz $^{3,14}$ has devised a semi-empirical scheme to systematize the observed behaviors. However, so far no fundamental explanations for the latter have been offered. CWST was applied with great elegance and one might say virtuosity in the above systems and appears to fit the data extraordinarily well. ${ }^{2,4,10} \mathrm{We}$, however, explain the apparent good fits as being due to layers of analysis that introduce a sufficient number of adjustable parameters to fit almost any magnetization curve. We think that the simple behaviors actually displayed by $\mathrm{CrCl}_{3}$ and the fluoride systems, as well as other simple magnetic systems, ${ }^{14}$ herald the need for a review of the validity of conventional spin-wave theory.

${ }^{1}$ R. J. Birgeneau, H. J. Guggenheim, and G. Shirane, Phys. Rev. B 1, 2211 (1970); 8, 304 (1973).

${ }^{2}$ H. W. de Wijn, L. R. Walker, and R. E. Walstedt, Phys. Rev. B 8, 285 (1973).

${ }^{3}$ N. Bykovetz, Ph.D. dissertation (University of Pennsylvania, 1976). To obtain the NMR data of Refs. 2 and 4, address requests to the original authors. Alternatively, email N. Bykovetz at nickbykovetz@ comcast.net.

${ }^{4}$ A. F. M. Arts and H. W. de Wijn, Phys. Rev. B 15, 4348 (1977).

${ }^{5}$ C. M. van Uijen E. Frikkee, and H. W. de Wijn, Phys. Rev. B 19, 509 (1979).

${ }^{6}$ A. Taroni, S. T. Bramwell, and P. C. W. Holdsworth, J Phys. Condens. Matter 20, 275233 (2008).

${ }^{7}$ H. Kubo, K. Shimohigashi, and I. Yamada, J. Phys. Soc. Jpn. 34, 1687 (1973).

${ }^{8}$ K. Hirakawa and H. Ikeda, J. Phys. Soc. Jpn. 35, 1328 (1973).

${ }^{9}$ W. Kleemann and F. J. Schafer, J. Magn. Magn. Mater. 21, 143 (1980).

${ }^{10}$ A. Narath and H. L. Davis, Phys. Rev. 137, A163 (1965).

${ }^{11}$ H. Kubo et al., J. Phys. Soc. Jpn. 42, 484 (1977).

${ }^{12}$ L. J. De Jongh, W. D. van Amstel, and A. R. Miedema, Physica 58, 277 (1972).

${ }^{13}$ S. I. Ben-Abraham and A. Nudelman, Phys. Rev. B 60, 11887 (1999).

${ }^{14}$ N. Bykovetz et al., J. Appl. Phys. 107, 09E142 (2010). 